\title{
MicroRNA-122a as a non-invasive biomarker for HCV genotype 4-related hepatocellular carcinoma in Egyptian patients
}

\author{
Eman G. E. El-Ahwany ${ }^{1}$, Lobna Mourad ${ }^{2}$, Mona M. K. Zoheiry ${ }^{1}$, Hoda Abu-Taleb ${ }^{3}$, Marwa Hassan ${ }^{1}$, \\ Raafat Atta ${ }^{4}$, Moataz Hassanien ${ }^{4}$, Suher Zada²
}

1Department of Immunology, Theodor Bilharz Research Institute, Giza, Egypt
2Department of Biology, The American University, Cairo, Egypt
${ }^{3}$ Department of Environmental Research, Theodor Bilharz Research Institute, Giza, Egypt
${ }^{4}$ Department of Hepatogastroenterology, Theodor Bilharz Research Institute, Giza, Egypt

Submitted: 5 August 2017

Accepted: 22 October 2017

Arch Med Sci 2019; 15 (6): 1454-1461

DOI: https://doi.org/10.5114/aoms.2019.86621

Copyright (c) 2019 Termedia \& Banach

\section{Abstract}

Introduction: Hepatitis C virus (HCV) infection persists in most infected individuals and can lead to the development of chronic hepatitis, cirrhosis and hepatocellular carcinoma (HCC). MicroRNAs (miRNAs) have a crucial role in various liver diseases, especially HCC. The expression profiles of circulating microRNAs have been studied aiming at the identification of novel non-invasive biomarkers. This study aims to develop a non-invasive diagnostic tool based on measuring the serum levels of different miRNAs in order to detect HCV-induced HCC at the early stages of the disease.

Material and methods: Five main miRNAs (miRNA-122a, miRNA-125a, miRNA-139, miRNA-145, and miRNA-199a) were selected according to the literature that demonstrated their unique expression pattern during HCC development. Serum samples were collected from 42 cases of chronic hepatitis C (CHC) without cirrhosis, 45 cases of $\mathrm{CHC}$ with cirrhosis (LC), 38 cases of HCC with HCV, and 40 healthy individuals serving as a control. The five miRNAs were measured using real-time reverse transcription PCR. The conventional HCC markers $\alpha$-fetoprotein (AFP) and des- $\gamma$-carboxyprothrombin (DCP) were measured with commercial kits.

Results: Serum levels of miRNA-122a, miRNA-125a, miRNA-139, miRNA-145, and miRNA-199a were significantly lower $(p<0.01)$ in HCC than in CHC and LC groups. As a single marker, miRNA-122a had the highest sensitivity for HCC, followed by miRNA-199a, miRNA-145, miRNA-139, and miRNA-125a. Conclusions: These findings indicate that measurement of serum levels of miRNA-122a, miRNA-125a, miRNA-139, miRNA-145, and miRNA-199a can differentiate HCC from CHC and LC. Our results suggest that serum miR-122 might serve as a novel and potential noninvasive biomarker for HCV-induced HCC.

Key words: chronic hepatitis C, microRNAs, hepatocellular carcinoma, serum biomarkers.

\section{Introduction}

Hepatocellular carcinoma (HCC) accounts for $90 \%$ of primary liver cancers and it represents the third most common cause of death from cancer worldwide, with an increasing incidence expected in the next decades [1]. The major risk factors are chronic viral hepatitis B and C (HBV

\author{
Corresponding author: \\ Marwa Hassan MD \\ Department of Immunology \\ Theodor Bilharz \\ Research Institute \\ Warrak El-Haddar \\ 12411 Giza, Egypt \\ E-mail: marwahassan_777@ \\ yahoo.com
}


and HCV), alcohol abuse, primary biliary cirrhosis, xenobiotics, diabetes, non-alcoholic fatty liver disease and genetic disorders such as hemochromatosis and $\alpha 1$-antitrypsin deficiency [2, 3]. To date, surgery remains the most effective treatment with curative potential. However, only about $10 \%$ to $20 \%$ of patients with HCC are currently eligible for surgical intervention [4]. Therefore, it is urgently required to find a sensitive biomarker to detect liver cancer at the early stage. Egypt has the highest prevalence of HCV in the world, estimated nationally at $14.7 \%$ [1], with genotype 4 being the most common [2]. Liver cancer development is thought to develop in a multi-step process requiring the accumulation of several structural and genomic alterations, thus affecting many different molecular pathways [5].

MicroRNAs (miRNAs) are an important class of small non-coding RNA molecules that have recently come to prominence as critical regulators in a wide array of mechanisms of cell physiology. There is increasing evidence that miRNAs may also have an important function in viral replication and may be used by host cells to control viral infection [6].

Cellular miRNAs may recognize viral RNAs and silence them [7]. It has been suggested that many of the miRNA changes that take place during hepatocarcinogenesis do so early; therefore, many changes that predispose to liver cancer have already taken place during liver cirrhosis and other premalignant lesions [8]. It was found that specific circulating miRNAs could be detected significantly earlier after liver injury. In 2006, it was first reported that down-regulation of miR-122 could be a potential biomarker for liver cancer in rodent and human HCC [9].

MiRNAs are not only found inside cells but they are also circulating in serum and other bodily fluids. The function of these extracellular circulating miRNAs is not well understood yet. They may be released due to tissue damage as in the case of miR-122 and miR-192, which is indicative of liver degeneration in mice in response to drug treatment [10]. They also act as mediators of cell-cell communication as seen in Epstein-Barr virus infected cells where miRNAs secreted by infected cells are taken up by monocyte-derived dendritic cells repressing certain target genes in the dendritic cells [11]. Changes in circulating miRNA profiles have been associated with various diseases. They may be used as potential biomarkers because of their stability in body fluids, sensitive detection by quantitative polymerase chain reaction (PCR), and non-invasiveness in obtaining samples of body fluids [12]. For example, serum miR-21, miR-122 and miR-223 were seen to be elevated in patients with HCC or chronic hepatitis, which means that these miRNAs could be used as diagnostic biomarkers for liver injury [13].

Although miRNA-based diagnostics has been successfully utilized to diagnose, monitor, and/or manage a wide range of neoplastic and non-neoplastic disorders, several knowledge-based and practical issues hamper their translation into routine clinical practice. These issues include lack of collaborative research efforts, miRNA sequence database errors, non-standardization of sample processing protocols, analytical variation, suboptimal RNA extraction methods, lack of an accurate and valid normalization protocol, non-reliability and non-reproducibility of many results, detection assays variability, and lack of cellular and clinical database resources [14].

Thus, the aim of the current work was to develop a non-invasive reliable diagnostic biomarker through a carefully designed study based on measuring the serum levels of different miRNAs in order to detect HCV genotype 4-induced HCC at the early stages of the disease.

\section{Material and methods}

This study involved 165 patients admitted to the Hepato-Gastroenterology Department, Theodor Bilharz Research Institute (TBRI), Giza, Egypt for evaluation of their HCV-related chronic liver disease. All had circulating anti-HCV genotype 4 antibodies, and no serologic evidence of co-infection with HBV. Patients were excluded from the study if they had parasitological, serological, histopathological, or ultrasonographic findings indicative of other etiologies of chronic liver disease (CLD) other than HCV, such as non-alcoholic steatohepatitis, autoimmune hepatitis, biliary disorders, malignancies other than HCC, bacterial infection, Schistosoma infection, or dual chronic hepatitis $B$ and $C$ infection.

All patients gave informed consent prior to inclusion in the study in conformance with the guidelines of the 1975 Declaration of Helsinki as reflected by approval of the institution's human research ethics committee. All procedures were medically indicated for patient management. Clinically and biochemically healthy, roughly age- and gender-matched persons $(n=40)$ served as control subjects.

The patients were subjected to thorough clinical examination and were assessed by laboratory investigations: ultra-sonography and liver biopsy using ultrasound-guided Menghini needle. HCC diagnosis is made upon the presence of hepatic lesion suggestive of malignancy by abdominal ultrasonography and triphasic abdominal computed tomography (CT) scan and confirmed to be HCC by histologic assessment of liver biopsy.

\section{Histopathologic study}

Assessment of grade of inflammation and stage of fibrosis was carried out in $5 \mu \mathrm{m}$ thick serial sections of formalin-fixed, paraffin-embedded blocks, stained with hematoxylin/eosin and Mas- 
son trichrome stains. The stage of hepatic fibrosis was determined according to the METAVIR scoring system (F0, F1, F2, F3 \& F4). We collected the F0, $F 1, F 2 \& F 3$ in one group, the chronic hepatitis $C$ (CHC) without cirrhosis and the F4 only in another group, the CHC with cirrhosis (LC) group.

\section{Laboratory investigations}

Liver function tests were done using commercially available kits. Hepatitis B markers were tested using enzyme immunoassay (EIA) kits (Axiom Diagnostics, Burstadt, Germany). Circulating anti-HCV (genotype 4) antibodies were detected using an EIA kit (Axiom Diagnostics, Burstadt, Germany), and the presence of HCV-RNA in patients' sera was detected by real-time PCR using the Amplicor test (Roche Diagnostic Systems; Meylan, France). Meanwhile, serum AFP was tested using the Eurogenetics EIA kit (Tessenderlo, Belgium).

\section{HCV detection}

Viral RNA was extracted using the QIAamp Viral RNA kit (Qiagen, Valencia, CA) and stored at $-80^{\circ} \mathrm{C}$. The serum HCV-RNA level was measured using One-Step Real-Time PCR according to manufacturer's protocol (Carlsbad, CA, USA).

\section{Serum preparation and RNA extraction}

For RNA isolation from serum, $250 \mu$ of serum was homogenized in $750 \mu \mathrm{l}$ of Trizol LS (Invitrogen, Carlsbad, CA, USA). Then $200 \mu$ l of chloroform was added to the sample and the mixed solution was centrifuged. After an additional chloroform extraction and precipitation with isopropanol, the pellet was washed twice by centrifugation with $70 \%$ ethanol. The RNA pellet was dried for $10 \mathrm{~min}$ at room temperature and dissolved in $30 \mu \mathrm{l}$ of diethylpyrocarbonate (DEPC)-treated water. DNase treatment (Qiagen, Valencia, CA) was carried out to remove any contaminating DNA. The concentration and quality of RNA were measured by A260/280 ratio and checked by gel electrophoresis individually.

Reverse transcription (RT) and quantitative PCR (qPCR)

RT and qPCR kits made specifically for accurate miRNA analysis (Applied Biosystems, Foster City, CA, USA) were used. For real-time PCR, $1.33 \mu \mathrm{l}$ of diluted RT products were mixed with $10 \mu \mathrm{l}$ of $2 \times$ TaqMan PCR master mixture, $1 \mu$ l of TaqMan MicroRNA assay and $7.67 \mu$ of nuclease-free water in a final volume of $20 \mu \mathrm{l}$ according to the manufacturer's instructions. All reactions were run on the ABI 7300 (Applied Biosystems, USA) using the following conditions: $95^{\circ} \mathrm{C}$ for $10 \mathrm{~min}$, followed by 40 cycles at $95^{\circ} \mathrm{C}$ for $15 \mathrm{~s}$, and $60^{\circ} \mathrm{C}$ for $1 \mathrm{~min}$. Real-time PCR was done in triplicate, includ- ing no-template controls. Relative expression of miRNA was calculated using the comparative cycle

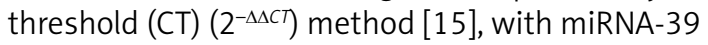
as the endogenous control to normalize the data.

\section{Statistical analysis}

Due to the magnitude and range of relative miRNA expression levels observed, results data were log transformed for analysis. Data are presented as mean \pm SE. There was no evidence against normality for the log transformed data as confirmed using the Kolmogorov-Smirnov test. Analysis of variance (ANOVA test) was used to evaluate expression differences of the chosen miRNAs between cases and controls.

Receiver operating characteristic (ROC) curves were constructed and the area under the curve (AUC) was calculated to evaluate the specificity and sensitivity of predicting cases and controls. All statistical tests were two-sided, and a probability level of $p<0.05$ was considered to be statistically significant. Data analysis was done using SPSS 20.0 software (IBM Corp.).

\section{Results}

Demographic, laboratory investigations, and clinical features of the patients

Clinically and biochemically healthy, roughly ageand gender-matched individuals $(n=40)$ served as a control population for patients with HCV-related liver fibrosis, cirrhosis, and primary HCC. Table I summarizes the epidemiological data of control subjects and patients, i.e. number, age, and gender. The biochemical parameters, i.e. aspartate aminotransferase (ASP), alanine aminotransferase (ALT), albumin, alkaline phosphatase (ALP), prothrombin concentration, $\alpha$-fetoprotein (AFP) and des- $\gamma$-carboxy prothrombin (DCP), were as expected, within the reference range for control subjects.

Serum HCV-RNA revealed that all patients were genotype 4. All the studied groups' patients had increased ALT, AST, albumin, ALP, prothrombin concentration, AFP and DCP $(p<0.001)$ compared to the control group and the AFP and DCP serum levels were increased significantly in HCC patients compared to hepatitis $C$ and cirrhosis patients $(p<0.001)$ (Table I).

\section{Serum expression levels of miRNA-122a, miRNA-125a, miRNA-139, miRNA-145 and miRNA-199a}

Serum levels of miRNA-122a, miRNA-125a, miRNA-139, miRNA-145 and miRNA-199a were significantly lower $(p<0.01)$ in HCC than in both CHC and LC groups. As a single marker, miRNA$122 \mathrm{a}$ had the highest sensitivity for HCC, followed 
Table I. Demographic and laboratory data of all studied groups

\begin{tabular}{|c|c|c|c|c|}
\hline Variables & $\begin{array}{l}\text { Controls } \\
(n=40)\end{array}$ & $\begin{array}{c}\text { CHC with early } \\
\text { fibrosis } \\
\begin{array}{c}\text { (F0 + F1 + F2 + F3) } \\
(n=42)\end{array}\end{array}$ & $\begin{array}{c}\text { CHC with cirrhosis } \\
\text { (F4) } \\
(n=45)\end{array}$ & $\begin{array}{c}\text { HCV-induced HCC } \\
(n=38)\end{array}$ \\
\hline Age & $47.0 \pm 3.5$ & $46.4 \pm 7.3$ & $51.3 \pm 4.7$ & $48.1 \pm 4.1$ \\
\hline Gender $(M: F)$ & $3: 1$ & $3: 2$ & $7: 5$ & $6: 4$ \\
\hline \multicolumn{5}{|l|}{ Liver function tests, mean \pm SD: } \\
\hline AST [U/I] & $21.32 \pm 1.07$ & $46.14 \pm 4.77^{\star}$ & $50.84 \pm 5.00^{\star}$ & $76.40 \pm 7.34^{\star \star}$ \\
\hline $\operatorname{ALT}[\mathrm{U} / \mathrm{l}]$ & $23.16 \pm 1.95$ & $44.12 \pm 5.50^{*}$ & $50.84 \pm 5.00^{\star}$ & $76.40 \pm 7.34^{\star *}$ \\
\hline $\operatorname{ALP}[U / I]$ & $189 \pm 41$ & $331 \pm 36^{*}$ & $336 \pm 48^{\star}$ & $420 \pm 33^{\star *}$ \\
\hline Albumin [g/dl] & $4.4 \pm 0.5$ & $3.60 \pm 0.74$ & $3.8 \pm 0.72$ & $3.08 \pm 0.48$ \\
\hline Prothrombin concentration & $95.6 \pm 3.4$ & $89.6 \pm 4.8$ & $41.5 \pm 11.1^{\star *}$ & $69.4 \pm 3.7^{\star}$ \\
\hline AFP [IU/ml] & $3.12 \pm 0.08$ & $8.86 \pm 0.11^{*}$ & $10.11 \pm 0.11^{\star}$ & $55.18 \pm 0.44^{* *}$ \\
\hline $\mathrm{DCP}[\mathrm{mAU} / \mathrm{ml}]$ & $30.42 \pm 0.70$ & $121.49 \pm 0.59^{*}$ & $123.62 \pm 0.38^{\star}$ & $456.52 \pm 0.66^{\star \star}$ \\
\hline
\end{tabular}

Data are expressed as mean \pm standard deviations (SD), CHC - chronic hepatitis C, HCC - hepatocellular carcinoma, HCV-hepatitis C virus, normal range for ALT and AST is up to $40 \mathrm{IU} / \mathrm{l}$, normal range for $A L P$ is up to $250 \mathrm{U} / \mathrm{l}$, normal range for albumin is $3.5-5 \mathrm{~g} / \mathrm{dl}$, normal range for prothrombin concentration is $80-100 \%$, normal range for AFP is 0.1-9.6 IU/ml, $\mathrm{mAU} / \mathrm{ml}$ - milli-absorbance unit/ml, normal range for des- $\gamma$-carboxyprothrombin $(D C P) \leq 40 \mathrm{mAU} / \mathrm{ml}$, ${ }^{*} p<0.001$ significantly higher than control group, ${ }^{* *} p<0.001$ significantly higher than CHC and LC groups.

Table II. Real-time qPCR expression levels of microRNAs in serum of the different studied groups

\begin{tabular}{|c|c|c|c|c|}
\hline MiRNAs/groups & Control & $\begin{array}{c}\text { CHC with early fibrosis } \\
\text { (F0 + F1 + F2 + F3) } \\
(n=42)\end{array}$ & $\begin{array}{c}\text { CHC with cirrhosis } \\
\text { (F4) } \\
(n=45)\end{array}$ & $\begin{array}{c}\text { HCV-induced HCC } \\
(n=38)\end{array}$ \\
\hline miRNA-122a & $19.69 \pm 0.33$ & $545.83 \pm 0.79^{* *}$ & $520.94 \pm 0.77^{* *}$ & $16.13 \pm 0.38^{\mathrm{a}}$ \\
\hline miRNA-125a & $20.57 \pm 0.54$ & $96.01 \pm 4.36^{\star *}$ & $100.54 \pm 0.81^{\star *}$ & $29.96 \pm 0.57^{\star \star}$ \\
\hline miRNA-139 & $20.53 \pm 0.56$ & $94.63 \pm 0.38^{\star *}$ & $86.02 \pm 0.400^{* *}$ & $30.03 \pm 0.43^{\star *}$ \\
\hline miRNA-145a & $20.65 \pm 0.52$ & $85.31 \pm 0.53^{\star *}$ & $80.74 \pm 0.59^{\star *}$ & $20.64 \pm 0.57$ \\
\hline miRNA-199a & $80.23 \pm 0.72$ & $330.38 \pm 0.74^{* *}$ & $311.98 \pm 0.72^{* *}$ & $66.16 \pm 0.44^{a}$ \\
\hline
\end{tabular}

${ }^{* *} p<0.001$ significantly higher than control, ${ }^{a} p<0.001$ significantly lower than control.

by miRNA-199a, miRNA-145, miRNA-139 and miRNA-125a (Table II).

\section{Correlation of the different serum} microRNAs levels in HCC patients with AFP and DCP levels

The AFP and DCP are directly correlated with miR-139, miR-145, miR-199a, miR-122 and miR125a (Table III). The results revealed a positive correlation between serum levels of miR-145 and miR-139 in HCC patients. Moreover, the serum levels of miR-199 were positively correlated with miR-139 and miR-145. However, a reverse correlation between miR-122 and miR-125a was also observed (Table IV).

\section{ROC curve and $\mathrm{Cl}$ analysis}

Receiver operator characteristic curves (ROC) and confidence intervals $(\mathrm{Cl})$ of miR-122a, $-125 \mathrm{a}$, $-139,-145$ and -199 a were established to discriminate HCV-induced HCC from CHC. MiR-122a $(A U C=0.897 ; 95 \% \mathrm{Cl}: 0.841-0.953)$, miR-199a $(A U C=0.889 ; 95 \% \mathrm{Cl}: 0.843-0.955), \operatorname{miR}-125 \mathrm{a}$
Table III. Correlation of different serum microRNAs levels in HCC patients with AFP and DCP levels

\begin{tabular}{|lcc|}
\hline MiRNAs/tumor markers & AFP & DCP \\
\hline miR-122 & $r=-0.525$ & $r=-0.534$ \\
& $p<0.001$ & $p<0.001$ \\
\hline miR-125a & $r=-0.416$ & $r=-0.429$ \\
& $p<0.001$ & $p<0.001$ \\
\hline miR-145 & $r=-0.519$ & $r=-0.528$ \\
& $p<0.001$ & $p<0.001$ \\
\hline miR-139 & $r=-0.431$ & $r=-0.440$ \\
& $p<0.001$ & $p<0.001$ \\
\hline miR-199a & $r=-0.553$ & $r=-0.562$ \\
& $p<0.001$ & $p<0.001$ \\
\hline
\end{tabular}

$(A U C=0.780 ; 95 \% \quad C l: 0.683-0.878), \operatorname{miR}-139$ $(A \cup C=0.930 ; 95 \% \mathrm{Cl}: 0.885-0.975)$ and miR-145 (AUC $=963 ; 95 \% \mathrm{Cl}:$ 0.932-0.994) (Figure 1).

\section{Diagnostic performance of circulating microRNAs in predicting HCC}

To evaluate the usefulness of the studied serum biomarkers for predicting significant HCC, the area under the sensitivity, specificity, positive 
Table IV. Correlation between different serum microRNA levels in HCC patients

\begin{tabular}{|lccccc|}
\hline MicroRNAs & miR-139 & miR-145 & miR-199 & miR-122 & miR-125 \\
\hline miR-139 & 1 & & & & \\
\hline miR-145 & $\begin{array}{l}r=0.988 \\
p<0.001\end{array}$ & 1 & & & \\
\hline miR-199 & $\begin{array}{l}r=0.987 \\
p<0.001\end{array}$ & $\begin{array}{c}r=0.994 \\
p<0.001\end{array}$ & 1 & & \\
\hline miR-122 & - & - & - & 1 & 1 \\
\hline miR-125 & - & - & - & $p<0.940$ & \\
& & & & & \\
\hline
\end{tabular}

Table V. Sensitivity and specificity of different microRNAs in predicting HCC

\begin{tabular}{|lcccc|}
\hline Variable(s) & Sensitivity & Specificity & PPV & NPV \\
\hline miRNA122 & 85.71 & 83.33 & 93.98 & 65.79 \\
\hline miRNA125a & 82.35 & 83.33 & 93.33 & 62.50 \\
\hline miRNA139 & 85.71 & 69.44 & 86.75 & 67.57 \\
\hline miRNA145 & 88.64 & 75.76 & 90.70 & 71.43 \\
\hline miRNA199a & 87.80 & 65.79 & 84.71 & 71.43 \\
\hline
\end{tabular}

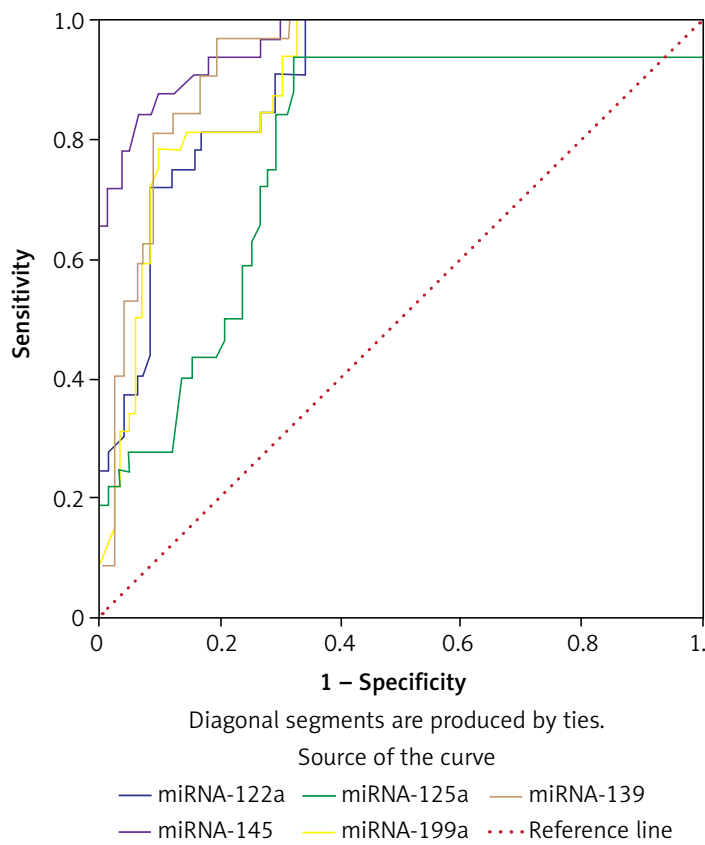

Figure 1. Receiver operator characteristic (ROC) curve analysis displaying diagnostic power of the studied miRNAs in the hepatocellular cancer (HCC) group

predictive value (PPV) and negative predictive value (NPV) were analyzed for each microRNA (Table V).

\section{Discussion}

Egypt has the highest prevalence of HCV infection in the world, estimated nationally as $14.7 \%$, with genotype 4 being the most common [16].
Hepatocellular carcinoma is still an extremely poor prognostic cancer that remains one of the most widespread and aggressive human malignancies worldwide. Uncontrolled cell growth and neoangiogenesis are key features of occurrence, invasion, metastasis and malignant progression of HCC $[17,18]$. The early diagnosis of HCC is of great clinical desirability since it promises good prognosis if the patients could get early surgical treatment. Until now, AFP has been mainly used clinically for the diagnosis of primary HCC; however, its sensitivity and specificity are not satisfactory [19]; therefore, novel biomarkers for early HCC diagnosis are gravely needed.

Results from recent studies revealed that circulating miRNAs are potential diagnostic biomarkers and prognostic factors in various kinds of diseases, especially in the field of human carcinogenesis. Chen et al. [20] demonstrated that by exploiting serum directly or by extracting RNA from the serum, it is possible to identify unique miRNA expression profiles specific for lung cancer, colorectal cancer and diabetes patients compared with healthy subjects.

In the past few years, miRNAs have been under the spotlight in the field of molecular oncology for their ability to be used as a powerful diagnostic tool.

Though several research groups have studied the role miRNAs play in the process of HCC development, the majority of the reports have studied histological specimens, therefore presenting limited clinical significance. Since miRNAs are shown to be stable in circulation, we propose that they can be utilized as a powerful non-invasive diagnostic tool for HCC. Therefore, we analyzed the expression profiles of five miRNAs (miRNA-122a, miRNA-125a, miRNA-139, miRNA-145, and miRNA-199a) in 165 individuals, and assessed their diagnostic ability compared to AFP and other conventional HCC markers. All patients had HCV genotype 4 since the viral genotype has an impact on miRNAs expression pattern as it has been previously described that many miRNAs were significantly different between CHC patients with HCV genotype 1 (GT-1) and non-genotype-1 (non-GT-1) [21]. 
The expression of miRNA-122a is specific to the liver, in which it is considered to represent $70 \%$ of the entire miRNA expression in the liver. Current studies have shown that the expression levels of miRNA-122a have declined during the process of hepatocarcinogenesis; hence miRNA-122a can function as a tumor suppressor. Our findings were consistent with previous studies, in which we observed a decline in the levels of miRNA-122a in HCC patients compared to the control individuals [22-24]. On the other hand, the levels of miRNA-122a increased significantly in the $\mathrm{CHC}$ and LC groups, suggesting that during the process of hepatocyte injury miRNA-122a levels increase drastically, and then decline back again significantly after the liver has entered carcinogenesis. According to a study by Jiang et al. [25], the serum levels of miRNA-122a have been shown to be elevated in HCC patients compared to healthy individuals. Also, as presented in our results, the ROC curve has demonstrated that out of the five miRNAs investigated, miRNA-122a has the highest specificity and sensitivity, making it an eligible candidate as a liver tumor marker. We inferred that the high AUC values are due to the fact that the patients in our studies had late stage HCC.

Halász et al. [26] have shown that in stage F4 fibrosis, the level of miR-122 was reduced as compared to stage FO, in which miR-122 showed a negative correlation with fibrosis stage in fibrotic liver samples and with LS values. These findings are supported by studies that discovered a negative correlation between miR-122 and fibrosis stage in chronic HCV infection, HCV-based HCC, and cirrhosis [27], in addition to the observations of a diminished level of miR-122 in non-alcoholic fatty liver disease (NAFLD) [28] and in HCC studies [29].

Loss of miR-122 appears to be a frequent event in HCC [30], which correlates with migration, invasion and in vivo tumorigenesis, whereas HCC cells expressing miR-122 maintain an epithelial phenotype [31]. MiR-122 is considered as a differentiation marker for hepatocytes [32], and a lower level of miR-122 might also reflect a lower degree of differentiation in the embryonal component. In addition, reduced expression of miR-122 is essential for the normal hepatocyte function; it positively regulates cholesterol, triglyceride accumulation, and fatty acid metabolism [33], constituting 70\% of the total miRNA pool of the liver.

The majority of studies that focused on the expression profiles of miRNA in HCC have shown that in most cases the progression of the malignancy is correlated with the down-regulation of the miRNAs. However, it needs to be taken into consideration that at the post-transcriptional level, miRNAs regulate hundreds of targets that are part of many signal transduction pathways, which makes the role of miRNAs in the process of hepatocarcinogenesis very complicated. According to different studies, it has been shown that miR$125 \mathrm{a}$ is down-regulated in a number of tumors, such as medulloblastoma, breast cancer, lung cancer, gastric cancer, and ovarian cancer. Bi et al. [34] showed that the expression of miR-125a is lower in HCC malignant tissue compared to non-tumor adjacent liver tissue and that the low expression level of miR-125a was associated with the progression of the disease as well as poor prognosis. In addition to the lower expression observed in tissues, also low expression of miR-125a was seen in HCC cell lines. In fact, it has been shown that the in vitro and in vivo ectopic expression of miR-125a can lead to inhibition of proliferation, migration, and invasion of the HCC cells [34]. Unlike what the literature and other studies have shown, the expression level of miR-125 in our study was slightly higher in the HCC patient samples compared to the control group. On the other hand, very high expression levels of miR-125a were observed in the $\mathrm{CHC}$ and $\mathrm{LC}$ patient groups.

As with miR-125a, according to previous studies the expression level of miR-139 has been shown to be significantly lower in HCC patient samples compared to control individuals. MiR-139 was shown to play an important role in hepatocarcinogenesis. In fact, high expression levels of miR139 were shown to inhibit cellular proliferation and invasion in malignant liver cells. In addition, in miR-139-transfected cells, apoptosis was shown to be highly induced, which suggests that miR139 can act as a tumor suppressor through the inhibition of cell proliferation and invasion in HCC [35]. As seen in our results, the expression levels of miR-139 were higher in the HCC patient group compared to the control group, which contradicts what has been reported in other studies, while the $\mathrm{CHC}$ and LC patient groups showed a significant increase in miR-139 expression. As a matter of fact, a possible explanation for the elevated expression levels for miR-125a and miR-139 in the HCC patient group is that HCV infection is the underlying cause of the HCC cases in the study, which might have an effect on the expression level of the two miRNAs. Although according to the literature, HCV infection should not affect the expression levels after the patient develops HCC, no study has examined that with HCV genotype 4. All patients in our study have developed HCC as a result of a genotype $4 \mathrm{HCV}$ infection, which may have resulted in the high expression level of miR$125 \mathrm{a}$ and miR-139, contradicting other studies.

MiR-145 was another miRNA tested among the miRNA panel investigated, in which its expression level showed no difference between the normal controls and the HCC patient group. As with the 
rest of the miRNAs investigated, miR-145 in the $\mathrm{CHC}$ and LC groups showed a significant increase compared to the control and HCC groups. According to Wang et al. [36], miR-145 was significantly down-regulated in HCC patient samples as well as HCC cell lines. As a matter of fact, the down-regulation of miR-145 was strongly linked to intrahepatic metastasis, tumor size, vascular invasion, and tumor grade, all suggesting that miR-145 acts as a tumor suppressor and its decreased expression results in progression of hepatocarcinogenesis.

The last miRNA we investigated was miR-199a, which was down-regulated in the HCC patient group compared to the control group, while it was significantly upregulated in the CHC and LC groups. The down regulation of miR-199a has been reported in several studies; in fact, miR-199a has been one of the most consistently reported miRNAs to be involved in HCC. MiR-199a is the third highly expressed miRNA in the liver, and in the case of HCC, it has been shown to be down-regulated especially in patients with HCV, HBV infections, and alcohol abuse [37]. The down-regulation of miR-199a in HCC patients has been associated with poor prognosis. The identification of miRNAs associated with HCC is crucial to developing new diagnostic and therapeutic tools with high specificity and sensitivity in order to combat this vicious human cancer.

In conclusion, our results indicate that measurement of serum levels of miRNA-122a, miRNA-125a, miRNA-139, miRNA-145 and miRNA-199a can help to differentiate HCC from CHC and LC. Also, they suggest that serum miR-122 might serve as a novel potential non-invasive biomarker for HCV-induced HCC.

\section{Acknowledgments}

This work was kindly supported and funded by the internal project, NO: 86 diagnosis, TBRI.

\section{Conflict of interest}

The authors declare no conflict of interest.

\section{References}

1. Parkin DM, Bray F, Ferlay J, Pisani P. Global cancer statistics. CA Cancer J Clin 2005; 55: 74-108.

2. Thorgeirsson SS, Grisham JW. Molecular pathogenesis of human hepatocellular carcinoma. Nat Genet 2002; 31: 339-46.

3. Li J, Huang Z, Wei L. Bioinformatics analysis of the gene expression profile of hepatocellular carcinoma: preliminary results. Contemp Oncol (Pozn) 2016; 20: 20-7.

4. Ji JF, Shi J, Budhu A, et al. MicroRNA expression, survival, and response to interferon in liver cancer. $\mathrm{N}$ Engl J Med 2009; 361: 1437-47.

5. Thorgeirsson SS, Grisham JW. Molecular pathogenesis of human hepatocellular carcinoma. Nat Gen 2002; 31 $339-46$.
6. Grassmann R, Jeang KT. The roles of microRNAs in mammalian virus infection. Biochem Biophys Acta 2008; 1779: 706-11.

7. Obbard DJ, Gordon KH, Buck AH, Jiggins FM. The evolution of RNAi as a defense against viruses and transposable elements. Philos Trans R Soc Lond B Biol Sci 2009; 364: 99-115.

8. Jiang J, Gusev Y, Aderca I, et al. Association of microRNA expression in hepatocellular carcinomas with hepatitis infection, cirrhosis, and patient survival. Clin Cancer Res 2008; 14: 419-27.

9. Kutay H, Bai S, Datta J, et al. Downregulation of miR-122 in rodent and human hepatocellular carcinomas. J Cell Biochem 2006; 99: 671-8.

10. Mitchell PS, Parkin RK, Kroh EM, et al. Circulating microRNAs as stable blood-based markers for cancer detection. Proc Natl Acad Sci USA 2008; 105: 10513-8.

11. Chikawa D, Komatsu S, Konishi H, Otsuji E. Circulating microRNA in digestive tract cancers. Gastroenterology 2012; 142: 1074-8.

12. Lawrie CH, Gal S, Dunlop HM, et al. Detection of elevated levels of tumor associated microRNAs in serum of patients with diffuse large B-cell lymphoma. $\mathrm{Br}$ J Heametol 2008; 141: 672-5.

13. Roderburg C, Luedde T. Circulating microRNAs as markers of liver inflammation, fibrosis and cancer. J Hepatol 2014; 61: 1434-7.

14. Shalaby T, Fiaschetti G, Baumgartner M, Grotzer MA. MicroRNA signatures as biomarkers and therapeutic target for CNS embryonal tumors: the pros and the cons. Int J Mol Sci 2014; 15: 21554-86.

15. Schmittgen TD, Livak KJ. Analyzing real-time PCR data by the comparative C(T) method. Nat Protoc 2008; 3: 1101-8.

16. Mahmoud YA, Mumtaz GR, Riome S, Miller D, Abu-Raddad LJ. The epidemiology of hepatitis C virus in Egypt: a systematic review and data synthesis. BMC Infect Dis 2013; 13: 288.

17. Qu Z, Jiang Y, Xu M, Lu MZ, Zhou B, Ding Y. Correlation of adrenomedullin with the erythropoietin receptor and microvessel density in hepatocellular carcinoma. Arch Med Sci 2015; 11: 978-81.

18. Chen G, Li X, Yang J, et al. Prognostic significance of cyclooxygenase-2 expression in patients with hepatocellular carcinoma: a meta-analysis. Arch Med Sci 2016; 12: 1110-7.

19. Zinkin NT, Grall F, Bhaskar K, et al. Serum proteomics and biomarkers in hepatocellular carcinoma and chronic liver disease. Clin Cancer Res 2008; 14: 470-7.

20. Chen X, Ba Y, Ma L, et al. Characterization of microRNAs in serum: a novel class of biomarkers for diagnosis of cancer and other diseases. Cell Res 2008; 18: 997-1006.

21. Chang CC, Lin CC, Hsieh WL, Lai HW, Tsai CH, Cheng YW. MicroRNA expression profiling in PBMCs: a potential diagnostic biomarker of chronic hepatitis C. Dis Markers 2014; 2014: 367157.

22. Luo J, Chen $M$, Huang $H$, et al. Circulating microRNA-122a as a diagnostic marker for hepatocellular carcinoma. Onco Targets Ther 2013; 6: 577-83.

23. Motawi TM, Sadik NA, Shaker OG, Ghaleb MH. Elevated serum microRNA-122/222 levels are potential diagnostic biomarkers in Egyptian patients with chronic hepatitis C but not hepatic cancer. Tumor Biol 2016; 7: 9865-74.

24. Ezzat H, Lotfy A, Alalfy M, et al. The significance of circulating Micro RNA-122 as a non-invasive diagnostic marker of liver injury in Egyptian chronic hepatitis C 
virus infected and cirrhotic patients with and without hepatocellular carcinoma. Clin Med Diagn 2014; 4: 1-8.

25. Jiang L, Cheng Q, Zhang B, Zhang M. Circulating microRNAs as biomarkers in hepatocellular carcinoma screening: a validation set from China. Medicine 2015; 94 : e603.

26. Halász T, Horváth G, Pár G, et al. miR-122 negatively correlates with liver fibrosis as detected by histology and FibroScan. World J Gastroenterol 2015; 21: 7814-23.

27. Marquez RT, Bandyopadhyay S, Wendlandt EB, et al. Correlation between microRNA expression levels and clinical parameters associated with chronic hepatitis C viral infection in humans. Lab Invest 2010; 90: 1727-36.

28. Lakner AM, Bonkovsky HL, Schrum LW. MicroRNAs: fad or future of liver disease. World I Gastroenterol 2011; 17: 2536-42.

29. Borel F, Konstantinova P, Jansen PL. Diagnostic and therapeutic potential of miRNA signatures in patients with hepatocellular carcinoma. J Hepatol 2012; 56: 1371-83.

30. Gyugos M, Lendvai G, Kenessey I, et al. MicroRNA expression might predict prognosis of epithelial hepatoblastoma. Virchows Arch 2014; 464: 419-27.

31. Karakatsanis A, Papaconstantinou I, Gazouli M, Lyberopoulou A, Polymeneas G, Voros D. Expression of microRNAs, miR-21, miR-31, miR-122, miR-145, miR-146a, miR-200c, miR-221, miR-222, and miR-223 in patients with hepatocellular carcinoma or intrahepatic cholangiocarcinoma and its prognostic significance. Mol Carcinog 2013; 52: 297-303.

32. Cairo S, Wang Y, de Reynies A, et al. Stem cell-like micro-RNA signature driven by Myc in aggressive liver cancer. Proc Natl Acad Sci USA 2010; 107: 20471-6.

33. Hu J, Xu Y, Hao J, Wang S, Li C, Meng S. MiR-122 in hepatic function and liver diseases. Protein Cell 2012; 3: 364-71.

34. Bi Q, Tang S, Xia L, et al. Ectopic expression of MiR-125a inhibits the proliferation and metastasis of hepatocellular carcinoma by targeting MMP11 and VEGF. PLoS One 2012; 7: e40169.

35. Gu W, Li X, Wang J. miR-139 regulates the proliferation and invasion of hepatocellular carcinoma through the WNT/TCF-4 pathway. Oncol Rep 2014; 31: 397-404.

36. Wang Y, Hu C, Cheng J, et al. MicroRNA-145 suppresses hepatocellular carcinoma by targeting IRS1 and its downstream Akt signaling. Biochem Biophys Res Commun 2014; 446: 1255-60.

37. Diaz G, Melis M, Tice A, et al. Identification of microRNAs specifically expressed in hepatitis C virus-associated hepatocellular carcinoma. Int J Cancer 2013; 133: 816-24. 